\title{
On the Geroch-Traschen class of metrics
}

\author{
R Steinbauer ${ }^{1}$ and J A Vickers ${ }^{2}$ \\ ${ }^{1}$ Fakultät für Mathematik, Universität Wien, Nordbergstrasse 15, A-1090 Wien, \\ Austria \\ ${ }^{2}$ School of Mathematics, University of Southampton, Highfield, Southampton SO17 \\ 1BJ, United Kingdom \\ E-mail: Roland.Steinbauer@univie.ac.at and J.A.Vickers@maths.soton.ac.uk
}

\begin{abstract}
We compare two approaches to Semi-Riemannian metrics of low regularity. The maximally "reasonable" distributional setting of Geroch and Traschen is shown to be consistently contained in the more general setting of nonlinear distributional geometry in the sense of Colombeau.
\end{abstract}

AMS classification scheme numbers: Primary: 83C75; secondary: 46T30, 53B30, 46F10, 46F30.

PACS numbers: $04.20 . \mathrm{Cv}, 04.20 . \mathrm{Dw}$, 02.30.Sa.

Submitted to: Class. Quantum Grav. 


\section{Introduction}

In this paper we deal with different approaches to metrics of low differentiability in general relativity. While normally relativity is formulated for smooth metrics, most of the relevant differential geometric results actually hold in the case where the metric is only locally $C^{2-}=C^{1,1}$, i.e., the first derivative being locally Lipschitz continuous. In particular, this condition directly gives unique (local) solvability of the geodesic equation. Moreover, by Rademacher's theorem the second derivatives are in $L_{\mathrm{loc}}^{\infty}$, hence the Riemann tensor can be regarded as a distribution.

When further lowering the differentiability of the metric one meets conceptual problems as one reaches the limits of classical (i.e., linear) distribution theory. Since Einstein's equations are nonlinear, one cannot simply pass from smooth solutions of the field equations to weak ones. In particular, the curvature tensor is only linear in the second derivatives of the metric but nonlinear in the lower order terms. Hence one cannot simply calculate the curvature from a general distributional metric.

In a classic paper [1] Geroch and Traschen studied the question under which minimal conditions on the metric one can compute the curvature. To be precise, they isolated a class of metrics - which we will refer to as gt-regular-for which on the one hand one may calculate the classical distributional curvature, and which on the other hand possesses a certain stability property. That is, they defined a notion of convergence for gt-regular metrics which implies the convergence of the respective curvature tensors in the class of distributions. Note that it is this stability property which makes it sensible to use gt-regular metrics to model singular matter configurations in relativity. A slightly more general class of metrics allowing for a distributional curvature tensor but lacking stability in the above sense was introduced by Garfinkle [2]. Finally, we also mention that the class of gt-regular metrics recently was rederived in a coordinate-free manner in [3], see also [4].

Although belonging to the Geroch-Traschen class is a sufficient condition to allow one to compute the distributional curvature, the question of necessity is more subtle. There are, however, indications that the gt-regular metrics form the largest "reasonable" class of distributional metrics: for example the only slightly more general Garfinkle class fails to be stable, while even for gt-regular metrics one cannot formulate the Bianchi identifies for example.

However Geroch and Traschen also proved that a gt-regular metric allows only for a limited range of concentration of the gravitating source: the curvature tensor of a gtregular metric is supported on a manifold of codimension at most one. This explicitly excludes many interesting scenarios, in particular, strings of matter and point particles.

In order to model a wider class of spacetimes some authors were lead to use alternative mathematical tools to describe space-times of low regularity. In particular, the theory of algebras of generalised functions due to J.F. Colombeau [5, 6, 7] proved to be useful in the context of cosmic strings [8, 9], Kerr-Schild geometries [10], and impulsive pp-waves $[11,12]$. Also it was used to study the initial value problem for 
the wave equation in conical space-times [9] and in singular space-times with locally bounded metrics [13]; for a recent overview see [14]. This approach goes beyond the limits of classical (linear) distribution theory - hence also beyond the class of gt-regular metrics - as it allows one to assign a product to an arbitrary pair of distributions. It is based upon regularising distributions via convolution and the use of asymptotic estimates in terms of a regularisation parameter. In many cases it also allows one to compare the result of a calculation in the algebra of generalised functions with classical distributions; this concept, called association, basically consists in looking at the weak limit as the regularisation parameter goes to zero.

In the case where we are given a gt-regular metric we therefore have two approaches at hand to compute the curvature: the classical distributional one due to Geroch and Traschen and the generalised function approach using Colombeau's construction. The natural question therefore arises as to whether these two approaches lead to the same answer. In this paper we give a complete and positive answer to this question. Along the way we prove several results on convergence of sequences of metrics generated via smoothing by convolution of gt-regular metrics which are of interest in their own right and provide refined stability results on the Geroch-Traschen class of metrics.

\section{Prerequisites}

In this section we introduce some notation and recall known material on linear and nonlinear distributional geometry to make the presentation self-contained. In particular, we define the notions of gt-regular as well as generalised metrics and collect some basic results on smoothings via convolution with strict delta nets.

We begin with some notational conventions. Throughout this paper $\Omega$ denotes an open subset of $\mathbb{R}^{n}$ and $M$ an oriented, smooth manifold of dimension $n$. Given two subsets $U$ and $V$ of $\Omega$ or of $M$ we use the notation $V \subset \subset U$ if the closure $\bar{V}$ of $V$ is still a subset of the interior $U^{\circ}$ of $U$. Moreover, $K$ and $L$ will always denote compact sets and $C$ will denote a generic constant.

\subsection{Linear distributional geometry}

The space of distributions on $M$ is the dual space (in the sense of the usual (LF)topology) of the space of compactly supported $n$-forms, i.e., $\mathcal{D}^{\prime}(M)=\left(\Omega_{c}^{n}(M)\right)^{\prime}$. Distributional sections of a vector bundle $E \rightarrow M$ over $M$ are defined as elements of the dual space of the compactly supported sections of $E^{*} \otimes \Lambda^{n}(M)$, where $E^{*}$ denotes the bundle dual to $E$ and $\Lambda^{n}(M)=T^{*} M \wedge \ldots \wedge T^{*} M$. Likewise distributional sections can be viewed as $\mathcal{C}^{\infty}$-linear maps from the sections of the dual bundle $\Gamma\left(E^{*}\right)$ to $\mathcal{D}^{\prime}(M)$ or as sections of $E$ with distributional, coefficients, that is we have

$\mathcal{D}^{\prime}(E):=\left(\Gamma_{c}\left(E^{*} \otimes \Lambda^{n}(M)\right)^{\prime} \cong L_{\mathcal{C}^{\infty}(M)}\left(\Gamma\left(E^{*}\right), \mathcal{D}^{\prime}(M)\right) \cong \mathcal{D}^{\prime}(M) \otimes_{\mathcal{C}^{\infty}(M)} \Gamma(E)\right.$.

The space of distributional tensor fields (tensor distributions) of type $(r, s)$ is denoted $\mathcal{D}_{s}^{\prime r}(M)$. There is a well-developed theory which parallels the smooth one but suffers 
from the natural limitations of distribution theory, e.g. in all multilinear operations only one factor may be distributional, while all others have to be smooth [15, 16]. For a pedagogical account see [17, Sec. 3.1].

Next we recall the definition of the (local) Sobolev spaces of integer order, i.e., for $m \in \mathbb{N}_{0}$ and $1 \leqslant p \leqslant \infty$ we set

$W^{m, p}\left(\mathbb{R}^{n}\right):=\left\{u \in \mathcal{D}^{\prime}\left(\mathbb{R}^{n}\right): \partial^{\alpha} u \in L^{p}\left(\mathbb{R}^{n}\right)\right.$ for all multi-indices with $\left.|\alpha| \leqslant m\right\}$

and denote the respective norms by \|\|$_{m, p}$. For any $\Omega$ we set

$W_{\text {loc }}^{m, p}(\Omega):=\left\{u \in \mathcal{D}^{\prime}(\Omega): \chi u \in W^{m, p}\left(\mathbb{R}^{n}\right)\right.$ for all test-functions $\left.\chi \in \mathcal{D}(\Omega)\right\}$.

Note that $u \in \mathcal{D}^{\prime}(\Omega)$ is in $W_{\text {loc }}^{m, p}(\Omega)$ iff on any open $V \subset \subset \Omega$ it agrees with a function in $W^{m, p}\left(\mathbb{R}^{n}\right)$. The space $W_{\text {loc }}^{m, p}(\Omega)$ is a Fréchet space with its topology induced by the family of semi-norms $p_{\chi}(u):=\|\chi u\|_{m, p}$ or alternatively by the \|\|$_{m, p^{-n o r m s}}$ on all relatively compact subsets $V$ of $\Omega$, which we denote by \|\|$_{W^{m, p}(V)}$.

On $M$ we define the local Sobolev spaces by means of local charts: denote by $\left(U_{\alpha}, \phi^{\alpha}\right)$ the charts of some atlas for $M$, then we set

$$
W_{\mathrm{loc}}^{m, p}(M):=\left\{u \in \mathcal{D}^{\prime}(M): \phi_{*}^{\alpha} u \in W_{\mathrm{loc}}^{m, p}\left(\phi^{\alpha}\left(U_{\alpha}\right)\right) \text { for all } \alpha\right\},
$$

where $\phi_{*}^{\alpha}$ denotes the push forward under the chart. $W_{\text {loc }}^{m, p}(M)$ is again a Fréchet space with its topology defined via the semi-norms of $\phi_{*}^{\alpha} u$ in $W_{\text {loc }}^{m, p}\left(\phi^{\alpha}\left(U_{\alpha}\right)\right)$, and one may show that this definition does not depend on the atlas. Finally, for $E \rightarrow M$ one defines the space of $W_{\text {loc }}^{m, p}$-sections likewise via vector bundle charts but for our purpose it will be sufficient to think of them as sections with $W_{\text {loc }}^{m, p}$-coefficients, i.e.,

$$
W_{\mathrm{loc}}^{m, p}(E)=W_{\mathrm{loc}}^{m, p}(M) \otimes_{\mathcal{C}^{\infty}(M)} \Gamma(E) .
$$

In case $p=2$ we use the usual convention and write $H_{\mathrm{loc}}^{m}$ for $W_{\text {loc }}^{m, 2}$ and in case $m=0$ we obtain the usual (local) Lebesgue spaces which we denote by $L_{\mathrm{loc}}^{p}$.

In [1] Geroch and Traschen defined the following class of metrics which we will call gt-regular.

Definition 2.1 (gt-regular metrics).

(i) We call a section of any vector bundle of regularity $H_{\mathrm{loc}}^{1} \cap L_{\mathrm{loc}}^{\infty}$ gt-regular.

(ii) A gt-regular metric $\mathbf{g}$ is a gt-regular section of $T_{2}^{0}(M)$ which is a Semi-Riemannian metric (of fixed index) almost everywhere.

The motivation for Geroch and Traschen to introduce this notion is that it follows from the coordinate definition that for a gt-regular metric it is possible to give a distributional definition of the Riemannian curvature tensor.

\subsection{Smoothings}

Next we recall the convergence properties of smoothing via convolution. The mollifiers we are going to use will be slightly more general than the standard ones obtained by scaling one fixed test-function with unit integral. More precisely we shall use.

Definition 2.2 (Smoothing with strict delta nets). 
(1) A net $\left(\psi_{\varepsilon}\right)_{\varepsilon \in(0,1]}$ of smooth functions on $\mathbb{R}^{n}$ is called a strict delta net, if

(i) $\operatorname{supp}\left(\psi_{\varepsilon}\right) \rightarrow\{0\}$ for $\varepsilon \rightarrow 0$

(ii) $\int \psi_{\varepsilon} \rightarrow 1$ for $\varepsilon \rightarrow 0$

(iii) $\psi_{\varepsilon}$ is uniformly bounded in $L^{1}$, i.e., $\exists C_{\psi}:\left\|\psi_{\varepsilon}\right\|_{L^{1}} \leqslant C_{\psi}$ for all $\varepsilon$ sufficiently small.

(2) For any strict delta net $\left(\psi_{\varepsilon}\right)_{\varepsilon}$ we denote by $d_{\psi_{\varepsilon}}$ the diameter of the support of $\psi_{\varepsilon}$, i.e., $d_{\psi_{\varepsilon}}:=\sup \left\{|x|: x \in \operatorname{supp}\left(\psi_{\varepsilon}\right)\right\}$.

(3) For any $f \in L_{\mathrm{loc}}^{1}(\Omega)$ we call the convolution $f_{\varepsilon}$ of $f$ with a strict a delta net $\left(\psi_{\varepsilon}\right)_{\varepsilon}$ a smoothing of $f$, i.e., for $x \in \Omega_{\psi_{\varepsilon}}:=\left\{y \in \Omega: \operatorname{dist}(y, \partial \Omega)>d_{\psi_{\varepsilon}}\right\}$ we set

$$
f_{\varepsilon}(x):=f * \psi_{\varepsilon}(x)=\int_{B\left(x, d_{\psi_{\varepsilon}}\right)} f(x-y) \psi_{\varepsilon}(y) d y,
$$

where $B(x, r)$ denotes the open ball of radius $r$ around $x$.

We recall the following results on smoothings (which are a mild generalisation of the ones found e.g. in $[18, \S 5.3, \S \mathrm{C} .4])$.

Lemma 2.3 (Smoothing via convolution). The smoothing of any $f \in L_{\mathrm{loc}}^{1}(\Omega)$ has the following properties.

(i) $f_{\varepsilon} \in \mathcal{C}^{\infty}\left(\Omega_{\psi_{\varepsilon}}\right)$ and $f_{\varepsilon} \rightarrow f$ almost everywhere.

(ii) If $f$ is continuous the convergence is actually uniform on compact subsets of $\Omega$.

(iii) If $f \in W_{\mathrm{loc}}^{m, p}(\Omega)$ for $1 \leqslant p<\infty$ then $f_{\varepsilon} \rightarrow f$ in $W_{\mathrm{loc}}^{m, p}(\Omega)$

Note that for $f \in L_{\mathrm{loc}}^{\infty}(\Omega)$ the last item implies $f_{\varepsilon} \rightarrow f$ in $L_{\mathrm{loc}}^{p}(\Omega)$ for all $p<\infty$ but not $p=\infty$. Indeed, the latter would contradict non-separability of $L^{\infty}$. For later reference we remark that also in this case $f_{\varepsilon}$ is nevertheless locally uniformly bounded. More precisely, we have for all $1 \leqslant p \leqslant \infty$ and all $f \in L_{\mathrm{loc}}^{p}(\Omega)$ that for any $V \subset \subset \Omega$

$$
\left\|f_{\varepsilon}\right\|_{L^{p}(V)} \leqslant\left\|\psi_{\varepsilon}\right\|_{L^{1}}\|f\|_{L^{p}(W)} \leqslant C_{\psi}\|f\|_{L^{p}(W)},
$$

where $W$ is any relatively compact subset of $\Omega$ with $V \subset \subset W$.

\subsection{Nonlinear distributional geometry}

In nonlinear distributional geometry [19], [17, Ch. 3] (in the sense of J.F. Colombeau[5, $6,7])$ one replaces the vector space $\mathcal{D}^{\prime}(M)$ of distributions by the algebra of generalised functions $\mathcal{G}(M)$ to overcome the problem of multiplication of distributions.

The basic idea of the construction is smoothing of distributions (via convolution) and the use of asymptotic estimates in terms of a regularisation parameter. The (special) Colombeau algebra of generalised functions on $M$ is defined as the quotient

$$
\mathcal{G}(M):=\mathcal{E}_{M}(M) / \mathcal{N}(M)
$$

of moderate nets of smooth functions modulo negligible ones, where the respective notions are defined by

$\mathcal{E}_{M}(M):=\left\{\left(u_{\varepsilon}\right)_{\varepsilon} \in \mathcal{C}^{\infty}(M): \forall K\right.$ compact $\left.\forall P \in \mathcal{P}(M) \exists N \in \mathbb{N}: \sup _{p \in K}\left|P u_{\varepsilon}(p)\right|=O\left(\varepsilon^{-N}\right)\right\}$ 
$\mathcal{N}(M):=\left\{\left(u_{\varepsilon}\right)_{\varepsilon} \in \mathcal{C}^{\infty}(M): \forall K\right.$ compact $\left.\forall P \in \mathcal{P}(M) \forall m \in \mathbb{N}: \sup _{p \in K}\left|P u_{\varepsilon}(p)\right|=O\left(\varepsilon^{m}\right)\right\}$

with $\mathcal{P}(M)$ denoting the space of linear differential operators on $M$. Elements of $\mathcal{G}(M)$ are denoted by $u=\left[\left(u_{\varepsilon}\right)_{\varepsilon}\right]=\left(u_{\varepsilon}\right)_{\varepsilon}+\mathcal{N}(M)$. With componentwise operations, $\mathcal{G}(M)$ is a fine sheaf of differential algebras where the derivations are Lie derivatives with respect to classical vector fields defined according to the formula $L_{X} u:=\left[\left(L_{X} u_{\varepsilon}\right)_{\varepsilon}\right]$. The spaces of moderate resp. negligible sequences and hence the algebra itself may be characterised locally, i.e., $u \in \mathcal{G}(M)$ iff $\phi_{*}^{\alpha} u \in \mathcal{G}\left(\phi^{\alpha}\left(U_{\alpha}\right)\right)$ for all charts $\left(U_{\alpha}, \phi^{\alpha}\right)$, where, on the open set $\phi^{\alpha}\left(U_{\alpha}\right) \subset \mathbb{R}^{n}$, partial derivatives replace differential operators in the respective estimates.

The $\mathcal{G}(M)$-module of generalised sections in $E \rightarrow M$ can be defined along the same lines using analogous asymptotic estimates. However, as in the case of $W_{\text {loc }}^{m p}$-valued sections it is more convenient to use the following algebraic description of generalised tensor fields

$$
\mathcal{G}(E)=\mathcal{G}(M) \otimes \Gamma(E)
$$

Hence generalised tensor fields are just given by classical ones with generalised coefficient functions. Moreover, we have the following chain of isomorphisms

$$
\mathcal{G}(E) \cong L_{\mathcal{C}^{\infty}(M)}\left(\Gamma\left(E^{*}\right), \mathcal{G}(M)\right) \cong L_{\mathcal{G}(M)}\left(\mathcal{G}\left(E^{*}\right), \mathcal{G}(M)\right)
$$

Spaces of generalised tensor fields will be denoted by $\mathcal{G}_{s}^{r}(M)$. Note that in contrast to classical distributions (c.f. (1)), generalised sections map generalised (and not merely smooth) sections of the dual bundle to generalised functions. It is precisely this property that allows one to raise and lower indices with the help of a generalised metric (see below) just as in the smooth setting.

Smooth functions are embedded into $\mathcal{G}(M)$ simply by the "constant" embedding $\sigma$, i.e., $\sigma(f):=\left[(f)_{\varepsilon}\right]$. On $\Omega$ compactly supported distributions are embedded into $\mathcal{G}$ via convolution with a mollifier $\rho \in \mathcal{S}\left(\mathbb{R}^{n}\right)$ with unit integral satisfying $\int \rho(x) x^{\alpha} d x=0$ for all $|\alpha| \geqslant 1$; more precisely setting $\rho_{\varepsilon}(x)=\left(1 / \varepsilon^{n}\right) \rho(x / \varepsilon)$, we have $\iota_{0}(w):=\left[\left(w * \rho_{\varepsilon}\right)_{\varepsilon}\right]$. (The fact that all moments of $\rho$ vanish is used to prove that $\left.\iota_{0}\right|_{\mathcal{C}^{\infty}}=\sigma$ in the quotient, which implies that the product of smooth functions is preserved in the constructiona distinguished feature of this approach, see below.) In case $\operatorname{supp}(w)$ is non-compact (hence $w * \rho_{\varepsilon}$ is not defined), one employs a sheaf-theoretic construction ([17, Sec. 1.1.2]) or alternatively uses an additional cut off at a different rate of growth (cf. [20]): We set $\psi_{\varepsilon}(x):=\chi(x / \sqrt{\varepsilon}) \rho_{\varepsilon}(x)$ where $\chi \in \mathcal{D}\left(B_{2}(0)\right), \chi=1$ on $B_{1}(0)$ and

$$
\mathcal{D}^{\prime}(\Omega) \ni w \mapsto \iota_{\psi}(w):=\left[\left(w * \psi_{\varepsilon}\right)_{\varepsilon}\right] \in \mathcal{G}(\Omega)(\varepsilon \text { small enough }),
$$

to obtain an embedding of distributions by convolution with a strict delta net which obviously commutes with derivatives. Note that this construction depends on the choice of the mollifier $\rho$ (but not on $\chi$ ), which allows for a flexible way of modelling singular objects. Moreover this construction can be lifted to $M$ decomposing $w$ via a partition of unity subordinate to the charts of an atlas and chartwise convolution (cf. [17, Thm. 3.2.10]). Such a procedure is, of course, dependent of the choice of charts and partition 
functions, hence non-geometric in an essential sense. There is, however, a version of the construction possessing a canonical and invariant embedding of $\mathcal{D}^{\prime}(M) \operatorname{resp} . \mathcal{D}_{s}^{\prime r}(M)$, the so-called full Colombeau algebras, see [21], resp. [22]. For the purpose of the present work it is, however, more convenient to use the (technically less demanding) special version: In fact we are going to derive convergence results for embedded distributions in the $W_{\text {loc }}^{m, p}$ - and $\mathcal{D}^{\prime}$-topologies respectively, which take place on (relatively) compact sets and so will be independent of the choice of charts, partition functions and also of $\rho$.

Finally, in light of Schwartz' impossibility result [23], the setting introduced above gives a minimal framework within which generalised sections of vector bundles, and, in particular, tensor fields may be subjected to nonlinear operations, while maintaining consistency with smooth and distributional geometry: tensor products of smooth sections are preserved as well as derivatives of distributional sections.

The interplay between generalised functions and distributions is most conveniently formalised in terms of the notion of association. A generalised function $u \in \mathcal{G}(M)$ is called associated to zero, $u \approx 0$, if one (hence any) representative $\left(u_{\varepsilon}\right)_{\varepsilon}$ converges to zero weakly. The equivalence relation $u \approx v: \Leftrightarrow u-v \approx 0$ gives rise to a linear quotient of $\mathcal{G}$ that extends distributional equality. Moreover, we call a distribution $w \in \mathcal{D}^{\prime}(M)$ the distributional shadow or macroscopic aspect of $u$ and write $u \approx w$ if, for all compactly supported $n$-forms $\boldsymbol{\nu}$ and one (hence any) representative $\left(u_{\varepsilon}\right)_{\varepsilon}$, we have

$$
\lim _{\varepsilon \rightarrow 0} \int_{M} u_{\varepsilon} \boldsymbol{\nu}=w(\boldsymbol{\nu}) .
$$

By (3), embeddings and association extend to generalised sections in a natural way.

Finally we recall the basic notions of Semi-Riemannian geometry in the generalised setting.

Definition 2.4 (Generalised metric). A symmetric section $\mathbf{g} \in \mathcal{G}_{2}^{0}(M)$ is called a generalised Semi-Riemannian metric if $\operatorname{det} \mathbf{g}$ is invertible in $\mathcal{G}\left(\mathrm{Vol}^{2}(M)\right)$, i.e., for any representative $\left(\operatorname{det}\left(\mathbf{g}_{\varepsilon}\right)\right)_{\varepsilon}$ of $\operatorname{det} \mathbf{g}$ we have

$$
\forall K \text { compact } \exists m \in \mathbb{N}: \inf _{p \in K}\left|\operatorname{det}\left(\mathbf{g}_{\varepsilon}(p)\right)\right| \geqslant \varepsilon^{m} .
$$

Here $\operatorname{Vol}^{q}(M)$ denotes the bundle of $q$-densities on $M$. The following characterisation of generalised metrics captures the intuitive idea of a generalised metric as a net of classical metrics approaching a singular limit: $\mathbf{g}$ is a generalised metric iff on every relatively compact open subset $V$ of $M$ there exists a representative $\left(\mathbf{g}_{\varepsilon}\right)_{\varepsilon}$ of $\mathbf{g}$ such that, for fixed $\varepsilon, \mathbf{g}_{\varepsilon}$ is a classical metric and its determinant, det $\mathbf{g}$, is invertible in the generalised sense. The latter condition basically means that the determinant is not too singular.

A generalised metric induces a $\mathcal{G}(M)$-linear isomorphism from $\mathcal{G}_{0}^{1}(M)$ to $\mathcal{G}_{1}^{0}(M)$. The inverse of this isomorphism gives a well-defined element of $\mathcal{G}_{0}^{2}(M)$, the inverse metric, which we denote by $\mathbf{g}^{-1}$, with representative $\left(\mathbf{g}_{\varepsilon}^{-1}\right)_{\varepsilon}$. The generalised covariant derivative, as well as the generalised Riemann, Ricci and Einstein tensors, of a 
generalised metric is defined by the usual formulae at the level of representatives. For further details see [24] and [17, Sec. 3.2].

\section{Notions of nondegeneracy}

In this short section we discuss some notions of nondegeneracy for metrics of low differentiability.

In the purely distributional setting, that is considering a metric as a symmetric element of $\mathcal{D}_{2}^{\prime 0}(M)$, one finds two different notions of nondegeneracy in the literature, i.e.,

(A) Marsden in [15] defines $\mathbf{g}$ to be nondegenerate if $\mathbf{g}(X, Y)=0$ for all smooth vector fields $X$ implies that the smooth vector field $Y$ vanishes.

(B) Parker in [16] defines $\mathbf{g}$ to be nondegenerate if it is nondegenerate off its singular support.

Note that notion (A) is strictly weaker than the usual pointwise condition. For example

$$
d s^{2}=x^{2} d x^{2}
$$

on $\mathbb{R}$ is nondegenerate in the sense of Marsden but is clearly not invertible on the whole of $\mathbb{R}$. On the other hand condition (B) does not put any restrictions on $g$ at the points where $g$ is not smooth. So the best option for a distributional metric would be to call it nondegenerate if both (A) and (B) hold.

On the other hand, in our view, the notion of nondegeneracy for gt-regular metrics was not unambiguously defined in [1]. The original statement saying that "the inverse of the metric exists everywhere" is mathematically best interpreted by saying that in the $L_{\text {loc }}^{\infty} \cap H_{\text {loc }}^{1}$-class of $\mathbf{g}$ there exists a representative which is invertible everywhere. This, however, would allow metric (4) to again count as nondegenerate: simply set the coefficient equal to 1 , for example, at $x=0$.

However, a natural notion of nondegeneracy for gt-regular metrics is available (see also [4]). Note that the space $H_{\mathrm{loc}}^{1} \cap L_{\mathrm{loc}}^{\infty}$ is actually an algebra. Indeed, $f g$ clearly is in $L_{\text {loc }}^{\infty} \subseteq L_{\text {loc }}^{2}$ and to show that $\partial_{j}(f g) \in L_{\text {loc }}^{2}$ we use the Leibnitz rule (which applies in all $\left.W_{\text {loc }}^{1, p}, p \geqslant 2\right)$ to write $\partial_{j}(f g)=\left(\partial_{j} f\right) g+\left(\partial_{j} g\right) f$ which is a sum of products $L_{\mathrm{loc}}^{2} \times L_{\mathrm{loc}}^{\infty}$ hence in $L_{\mathrm{loc}}^{2}$. Also a function $f \in H_{\mathrm{loc}}^{1} \cap L_{\mathrm{loc}}^{\infty}$ which is locally uniformly bounded away from zero, i.e., which satisfies

$$
\forall K \text { compact } \exists C_{K}:|f(x)| \geqslant C_{K}>0 \text { almost everywhere on } K \text {, }
$$

is invertible and $1 / f \in H_{\mathrm{loc}}^{1} \cap L_{\mathrm{loc}}^{\infty}$ is again locally uniformly bounded away from zero. Therefore we employ the following definition of nondegeneracy for gt-regular metrics (see also [3], p. 14).

Definition 3.1 (Nondegeneracy of gt-regular metrics).

We call a gt-regular metric $\mathbf{g}$ nondegenerate if its determinant is locally uniformly bounded away from zero, i.e.,

$$
\forall K \text { compact } \exists C_{K}:|\operatorname{det} \mathbf{g}(x)| \geqslant C_{K}>0 \text { almost everywhere on } K \text {. }
$$


Hence the determinant det $\mathbf{g}$ of a nondegenerate gt-regular metric $\mathbf{g}$ is an invertible density of regularity $H_{\mathrm{loc}}^{1} \cap L_{\mathrm{loc}}^{\infty}$ with (det $\left.\mathbf{g}\right)^{-1} \in H_{\mathrm{loc}}^{1} \cap L_{\mathrm{loc}}^{\infty}$ again locally uniformly bounded away from zero. Hence by the cofactor formula the inverse $\mathbf{g}^{-1}$ of $\mathbf{g}$ is again of regularity $H_{\mathrm{loc}}^{1} \cap L_{\mathrm{loc}}^{\infty}$ and nondegenerate in the sense that its $\operatorname{determinant} \operatorname{det}\left(\mathbf{g}^{-1}\right)$ is locally uniformly bounded away from zero.

However, this notion of nondegeneracy still does not have optimal stability properties with respect to smoothing via convolution and we will come back to discuss this issue in section 4.

To end this section we remark that the problems discussed above all originate from the fact that neither the distributional nor the gt-setting can provide pointwise resp. pointwise everywhere control on the metric. In contrast to this the condition of nondegeneracy employed for generalised metrics in Definition 2.4 allows for a pointwise control on generalised points as is shown in [17, Thm. 3.2.4].

\section{Smoothing gt-regular metrics}

In this section we provide a detailed account on stability properties of gt-regular metrics under smoothing with strict delta nets and of convergence results of embeddings of gtregular metrics into the Colombeau algebra.

We introduce the following notation: given a gt-regular metric $\mathbf{g}$ with local components $g_{i j}$ we will write $g_{i j}^{\varepsilon}$ for their smoothings, i.e., $g_{i j}^{\varepsilon}=g_{i j} * \psi_{\varepsilon}$, with $\left(\psi_{\varepsilon}\right)_{\varepsilon}$ being a strict delta net, and denote the resulting metric by $\mathbf{g}_{\varepsilon}$.

To begin with we collect some convergence results for products of nets of functions in $H_{\text {loc }}^{1} \cap L_{\text {loc }}^{\infty}$ generated by smoothing via convolution with strict delta nets. Given a function $f \in H_{\mathrm{loc}}^{1} \cap L_{\mathrm{loc}}^{\infty}$ we have from Lemma 2.3 (iii) that $f_{\varepsilon} \rightarrow f \in H_{\mathrm{loc}}^{1} \cap L_{\mathrm{loc}}^{p}$ for all $p<\infty$. Also given $f_{1}, \ldots, f_{m} \in H_{\mathrm{loc}}^{1} \cap L_{\mathrm{loc}}^{\infty}$ the product $f_{1} \cdots f_{m}$ is in $H_{\mathrm{loc}}^{1} \cap L_{\mathrm{loc}}^{\infty}$ and $\left(f_{1} \cdots f_{m}\right)_{\varepsilon}=\left(f_{1} \cdots f_{m}\right) * \psi_{\varepsilon} \rightarrow f_{1} \cdots f_{m}$ in $H_{\text {loc }}^{1} \cap L_{\text {loc }}^{p}$ for all $p<\infty$. We shall, however, be interested in convergence of curvature quantities derived from the componentwise smoothing of gt-regular metrics. Hence we have to study convergence properties of (derivatives of) $f_{1 \varepsilon} \cdots f_{m \varepsilon}$ rather than $\left(f_{1} \cdots f_{m}\right)_{\varepsilon}$.

Next we connect the products of nets of functions that arise in our approach to the general theory given in [25, Sec. II.7]. In the latter context the product we are dealing with is called strict product (of type (7.4)), that is, given two distributions $u$ and $v$ we look at the limit

$$
\lim _{\varepsilon \rightarrow 0}\left(u * \psi_{\varepsilon}\right)\left(v * \psi_{\varepsilon}\right) .
$$

If it exists for all strict delta nets $\left(\psi_{\varepsilon}\right)_{\varepsilon}$ (it is then automatically independent of the particular choice of $\psi_{\varepsilon}$ ) we call the limit the strict product of $u$ and $v$ and denote it by $[u v]$. The strict product can be placed in a hierarchy of products of distributions (see [25], p. 69) which are all compatible with the Colombeau product in the sense of association ([25], Prop. 10.3). 
Likewise we can make use of the $W_{\text {loc }}^{m, p}$-duality product, that is also contained in the above mentioned hierarchy. More precisely, one can define (by duality, [25], Prop. 5.2) a continuous product

$$
W_{\mathrm{loc}}^{m, q} \times W_{\mathrm{loc}}^{l, p} \rightarrow W_{\mathrm{loc}}^{k, r}
$$

if $l, m \in \mathbb{Z}$ with $l+m \geqslant 0,1 \leqslant p, q \leqslant \infty$ with $1 / p+1 / q \leqslant 1$ and $k, r$ are defined by $k:=\min (l, m), 1 / r:=1 / p+1 / q$. (For the spaces $W_{\text {loc }}^{m, p}$ for negative $k$ see e.g. [26, Ch. 3 - although we will not need to consider them in the following.) This product is more special than the strict product, although it is compatible with it, and has the additional benefit that it is partially associative, i.e., $(f u) v=u(f v)$ for all smooth $f$. We will have to deal with products containing many factors and so we have to be careful with the loss in $r$ in formula (8); only the special case $p=q=\infty$ does not share this problem but, on the other hand, this case lacks stability under smoothing as discussed below Lemma 2.3.

We now give a useful auxiliary result which (partially) follows from the general statements above and is needed to establish the results later in this section.

Lemma 4.1 (Convergence of products in $\left.H_{\mathrm{loc}}^{1} \cap L_{\mathrm{loc}}^{\infty}\right)$. Let $\left(\psi_{\varepsilon}\right)_{\varepsilon}$ be a strict delta net.

(i) If $g_{1}, \ldots g_{m} \in L_{\mathrm{loc}}^{\infty}(m \in \mathbb{N})$, then

$$
g_{1 \varepsilon} \cdots g_{m \varepsilon} \rightarrow g_{1} \cdots g_{m} \text { in } L_{\mathrm{loc}}^{p} \text { for all } p<\infty .
$$

(ii) If $f \in L_{\mathrm{loc}}^{p}$ with $1 \leqslant p<\infty$ and $\left(g_{\varepsilon}\right)_{\varepsilon}$ is a locally uniformly bounded net converging pointwise almost everywhere to some $g \in L_{\mathrm{loc}}^{\infty}$, then

$$
f_{\varepsilon} g_{\varepsilon} \rightarrow f g \text { in } L_{\mathrm{loc}}^{p}
$$

(iii) If $f_{1}, \ldots, f_{m} \in H_{\mathrm{loc}}^{1} \cap L_{\mathrm{loc}}^{\infty}(m \in \mathbb{N})$, then

$$
f_{1 \varepsilon} \cdots f_{m \varepsilon} \rightarrow f_{1} \cdots f_{m} \text { in } H_{\mathrm{loc}}^{1} \cap L_{\mathrm{loc}}^{p} \text { for all } p<\infty .
$$

Observe that statement (iii) says that the product of the smoothings of gt-regular functions converges in the same sense (i.e., in $H_{\mathrm{loc}}^{1} \cap L_{\mathrm{loc}}^{p}$ for all $p<\infty$ ) as the smoothing of (a product of) gt-regular functions.

Proof. (i) On any relatively compact set $V$ we have for all $p<\infty$

$$
\begin{aligned}
\left\|g_{1 \varepsilon} \cdots g_{m \varepsilon}-g_{1} \cdots g_{m}\right\|_{L^{p}(V)} & \leqslant \ldots+\left\|g_{1 \varepsilon} \cdots\left(g_{j \varepsilon}-g_{j}\right) \cdots g_{m}\right\|_{L^{p}}+\ldots \\
& \leqslant \ldots+\left\|g_{1 \varepsilon} \cdots g_{j-1 \varepsilon} g_{j+1} \cdots g_{m}\right\|_{L^{\infty}}\left\|g_{j \varepsilon}-g_{j}\right\|_{L^{p}}+\ldots .
\end{aligned}
$$

Now the respective first terms are bounded by estimate (2) and the convergence is due to Lemma 2.3(iii).

(ii) On any $V$ as above we write

$$
\left\|f_{\varepsilon} g_{\varepsilon}-f g\right\|_{L^{p}(V)} \leqslant\left\|g_{\varepsilon}\right\|_{L^{\infty}}\left\|f_{\varepsilon}-f\right\|_{L^{p}}+\left\|f\left(g_{\varepsilon}-g\right)\right\|_{L^{p}} .
$$

For the first term convergence follows from Lemma 2.3(iii) and the assumptions on $\left(g_{\varepsilon}\right)_{\varepsilon}$. To deal with the other term observe that

$$
f g_{\varepsilon} \rightarrow f g \text { in } L^{p}(V) .
$$


Indeed we have convergence almost everywhere by assumption as well as $\left|f(x) g_{\varepsilon}(x)\right| \leqslant$ $C|f(x)| \in L^{p}(V)$ almost everywhere. So dominated convergence applies to give the result.

(iii) $L_{\text {loc }}^{p}$-convergence for all $p<\infty$ follows from (i) and we only have to show $L_{\text {loc }}^{2}$ convergence of the derivatives. By the Leibnitz rule we have to show that for all $1 \leqslant j \leqslant n$ and all $1 \leqslant l \leqslant m$

$$
f_{1 \varepsilon} \cdots f_{l-1 \varepsilon}\left(\partial_{j} f_{l \varepsilon}\right) f_{l+1 \varepsilon} \cdots f_{m \varepsilon} \rightarrow f_{1} \cdots f_{l-1}\left(\partial_{j} f_{l}\right) f_{l+1} \cdots f_{m}
$$

in $L_{\text {loc }}^{2}$. This, however, follows from (ii) with $p=2$, since $f_{1 \varepsilon} \cdots f_{l-1 \varepsilon} f_{l+1 \varepsilon} \cdots f_{m \varepsilon}$ is a locally uniformly bounded net which by Lemma 2.3(i) converges pointwise almost everywhere to $f_{1} \cdots f_{l-1} f_{l+1} \cdots f_{m}$ and $\left(\partial_{j} f_{l \varepsilon}\right)=\left(\partial_{j} f_{l}\right)_{\varepsilon}$ is the smoothing of an $L_{\text {loc }}^{2}$ function.

We now obtain as a direct consequence of Lemma 4.1(iii) a stability result for the determinant of gt-regular metrics.

Proposition 4.2 (Stability of the determinant). Let $\mathbf{g}$ be a nondegenerate gt-regular metric and let $\left(\psi_{\varepsilon}\right)_{\varepsilon}$ be a strict delta net. Then we have for the determinant of the smoothing

$$
\operatorname{det}\left(\mathbf{g}_{\varepsilon}\right) \rightarrow \operatorname{det} \mathbf{g} \text { in } H_{\mathrm{loc}}^{1} \cap L_{\mathrm{loc}}^{p} \text { for all } p<\infty \text {. }
$$

In particular, we have for any embedding $\operatorname{det}(\iota(g)) \approx \operatorname{det}(g)$.

Next we discuss nondegeneracy of the smoothing of a nondegenerate gt-regular metric. Of course, the key is that the determinant of the smoothed metric has to be nonvanishing in an appropriate sense, which turns out to be a delicate matter: Unfortunately Proposition 4.2 does not give pointwise (let alone uniform) control on the determinant of the smoothing. Recall that such a condition will be needed to prove that the smoothing of a nondegenerate gt-regular metric is a generalised metric (cf. Definition 2.4-we will aim at proving this condition for $m=0$ ).

As a preparation we first discuss the scalar case. Suppose $f \in H_{\mathrm{loc}}^{1} \cap L_{\mathrm{loc}}^{\infty}$ is positive a.e. and locally uniformly bounded away from zero, i.e., satisfies (5). Then we know that $1 / f \in H_{\mathrm{loc}}^{1} \cap L_{\mathrm{loc}}^{\infty}$ and we want to secure that $1 / f_{\varepsilon} \rightarrow 1 / f$ in $H_{\mathrm{loc}}^{1} \cap L_{\mathrm{loc}}^{p}$ for all $p<\infty$. This will be achieved if $1 / f_{\varepsilon}$ is a uniformly bounded net on all relatively compact $V$ for small $\varepsilon$, which in turn is guaranteed by the following condition

$$
\forall K \text { compact } \exists C_{K}^{\prime} \exists \varepsilon_{0}(K): f_{\varepsilon}(x) \geqslant C_{K}^{\prime}>0 \forall x \in K, \forall \varepsilon \leqslant \varepsilon_{0}(K) \text {. }
$$

which gives uniform control on the positivity of the smoothing. Unfortunately this condition does not follow from (5) if we use arbitrary strict delta nets. (As an explicit counterexample take $f(x)=H(-x)+3 H(x)$ with $H$ denoting Heaviside's step function and use the strict delta net $\psi_{\varepsilon}(x)=1 / \varepsilon(2 \rho((x-\varepsilon) / \varepsilon)-\rho((x+\varepsilon) / \varepsilon))$, where $\rho$ is a standard bump function around zero with unit integral. Then $f_{\varepsilon}(0)=-1$ for all $\varepsilon$.)

Indeed to preserve positivity in the above sense during the smoothing (i.e., such that positivity and (5) imply (10)) one would, in a first attempt, use positive strict delta 
nets. However, recall that in the Colombeau approach it is essential to use mollifiers with vanishing moments and that such a mollifier cannot be positive. Nevertheless it is possible to provide strict delta nets which have vanishing moments and at the same time allow for good control on the $L^{1}$-norm of their negative parts, which is the essential ingredient for preserving positivity. More precisely we have the following result which we prove in the appendix.

Lemma 4.3 (Existence of admissible mollifiers). There exist strict delta nets $\left(\rho_{\varepsilon}\right)_{\varepsilon}$ with

(i) $\operatorname{supp}\left(\rho_{\varepsilon}\right) \subseteq B_{\varepsilon}(0)$ for all $\varepsilon \in(0,1]$

(ii) $\int \rho_{\varepsilon}(x) d x=1 \quad$ for all $\varepsilon \in(0,1]$

which are moderate, have finally vanishing moments and the negative parts have arbitrarily small $L^{1}$-norm, i.e., $\left(\rho_{\varepsilon}\right)_{\varepsilon}$ additionally satisfies

(iii) $\forall \alpha \in \mathbb{N}_{0}^{n} \exists p: \sup _{x \in \mathbb{R}^{n}}\left|\partial^{\alpha} \rho_{\varepsilon}(x)\right|=O\left(\varepsilon^{-p}\right)$

(iv) $\forall j \in \mathbb{N} \exists \varepsilon_{0}: \int x^{\alpha} \rho_{\varepsilon}(x) d x=0 \quad$ for all $1 \leqslant|\alpha| \leqslant j$ and all $\varepsilon \leqslant \varepsilon_{0}$

(v) $\forall \eta>0 \exists \varepsilon_{0}: \int\left|\rho_{\varepsilon}(x)\right| d x \leqslant 1+\eta$ for all $\varepsilon \leqslant \varepsilon_{0}$.

We will call strict delta nets $\left(\rho_{\varepsilon}\right)_{\varepsilon}$ as provided by Lemma 4.3 admissible mollifiers and from now on consider smoothings generated by convolution with such delta nets. Also, convolution with an admissible strict delta net provides an embedding $\iota_{\rho}$ of distributions in the Colombeau algebra as is shown in Corollary Appendix A.2 in the appendix.

We next show that smoothing with admissible mollifiers indeed preserves positivity in an appropriate sense.

Lemma 4.4 (Positive smoothing and convergence of the inverse). Let $f \in L_{\text {loc }}^{\infty}, f>0$ almost everywhere and locally uniformly bounded away from zero, i.e.,

$\forall K$ compact $\exists C_{k}: f(x) \geqslant C_{K}>0$ almost everywhere on $K$.

Then for any admissible mollifier $\left(\rho_{\varepsilon}\right)_{\varepsilon}$ we have.

(i) The smoothing $f_{\varepsilon}=f * \rho_{\varepsilon}(x)$ is a net, locally uniformly bounded away from zero, i.e.,

$$
\forall L \text { compact } \exists C_{L}^{\prime} \exists \varepsilon_{0}(L): f_{\varepsilon}(x) \geqslant C_{L}^{\prime}>0 \forall x \in L, \forall \varepsilon \leqslant \varepsilon_{0}(L) .
$$

(ii) For any open and relatively compact set $V$ there exists $\varepsilon_{0}(V)$ such that $1 / f_{\varepsilon}$ is a smooth and uniformly bounded net on $V$, i.e., $\left\|1 / f_{\varepsilon}\right\|_{L^{\infty}(V)} \leqslant C$ for all $\varepsilon \leqslant \varepsilon_{0}(V)$ and

$$
\frac{1}{f_{\varepsilon}(x)}=\frac{1}{f * \rho_{\varepsilon}(x)} \rightarrow \frac{1}{f} \text { in } L_{\mathrm{loc}}^{p} \text { for all } p<\infty .
$$

(iii) If, in addition, $f \in H_{\mathrm{loc}}^{1} \cap L_{\mathrm{loc}}^{\infty}$ then we can strengthen the convergence assertion to $\frac{1}{f_{\varepsilon}(x)}=\frac{1}{f * \rho_{\varepsilon}(x)} \rightarrow \frac{1}{f}$ in $H_{\mathrm{loc}}^{1} \cap L_{\mathrm{loc}}^{p}$ for all $p<\infty$. 
Note that although $1 / f \in H_{\mathrm{loc}}^{1} \cap L_{\mathrm{loc}}^{\infty}$ even if we drop the positivity assumption in (ii) and only ask for (5) the convergence result fails in general: such a function could change sign forcing the smoothing to attain a zero.

Proof. (i) Let $L$ be compact, $x \in L$ and choose $K$ compact such that $L \subset \subset K$. We split $\rho_{\varepsilon}$ into its positive and negative part (i.e., $\rho_{\varepsilon}=\rho_{\varepsilon}^{+}-\rho_{\varepsilon}^{-}$, with $\rho_{\varepsilon}^{+}:=\max \left(\rho_{\varepsilon}, 0\right)$, $\left.\rho_{\varepsilon}^{-}:=-\min \left(\rho_{\varepsilon}, 0\right)\right)$ to obtain

$$
f_{\varepsilon}(x)=f *\left(\rho_{\varepsilon}^{+}-\rho_{\varepsilon}^{-}\right)(x) \geqslant f * \rho_{\varepsilon}^{+}(x)-\left\|f * \rho_{\varepsilon}^{-}(x)\right\|_{L^{\infty}(L)} .
$$

Estimating the first term on the r.h.s. of (11) we have

$$
f * \rho_{\varepsilon}^{+}(x)=\int f(x-y) \rho_{\varepsilon}^{+}(y) d y \geqslant C_{K}\left\|\rho_{\varepsilon}^{+}\right\|_{L^{1}} \geqslant C_{K} .
$$

Using inequality (2) on the second term on the r.h.s. of (11) we obtain for $\varepsilon$ small enough

$$
\left\|f * \rho_{\varepsilon}^{-}\right\|_{L^{\infty}(L)} \leqslant\|f\|_{L^{\infty}(K)}\left\|\rho_{\varepsilon}^{-}\right\|_{L^{1}} \leqslant\|f\|_{L^{\infty}(K)} \frac{\eta}{2},
$$

where $\eta$ is the constant of Lemma 4.3(v). Combining the latter two estimates and choosing $\eta \leqslant C_{K} /\|f\|_{L^{\infty}(K)}$ we obtain

$$
f_{\varepsilon}(x) \geqslant C_{K}-\|f\|_{L^{\infty}(K)} \frac{\eta}{2} \geqslant \frac{C_{K}}{2}=: C_{L}^{\prime}>0 .
$$

(ii) Let $V$ be open and relatively compact. Then by (i) $1 / f_{\varepsilon} \in \mathcal{C}^{\infty}(V)$ form a uniformly bounded net for $\varepsilon$ small enough. Moreover, $f_{\varepsilon} \rightarrow f$ in $L_{\text {loc }}^{p}$ for all $p<\infty$ by Lemma 2.3(iii). So we find for all $p<\infty$

$$
\left\|\frac{1}{f_{\varepsilon}}-\frac{1}{f}\right\|_{L^{p}(V)} \leqslant\left\|\frac{1}{f_{\varepsilon} f}\right\|_{L^{\infty}}\left\|f-f_{\varepsilon}\right\|_{L^{p}} \leqslant \frac{1}{C_{\bar{V}} C_{\bar{V}}^{\prime}}\left\|f_{\varepsilon}-f\right\|_{L^{p}} \rightarrow 0 .
$$

(iii) In view of (ii) it remains to deal with the derivatives and we write for all $1 \leqslant j \leqslant n$

$$
\begin{aligned}
\left\|\partial_{j}\left(\frac{1}{f_{\varepsilon}}-\frac{1}{f}\right)\right\|_{L^{2}(V)} & =\left\|\frac{f^{2} \partial_{j} f_{\varepsilon}-f_{\varepsilon}^{2} \partial_{j} f}{f_{\varepsilon}^{2} f^{2}}\right\|_{L^{2}} \leqslant\left\|\frac{1}{f_{\varepsilon}^{2} f^{2}}\right\|_{L^{\infty}}\left\|f^{2} \partial_{j} f_{\varepsilon}-f_{\varepsilon}^{2} \partial_{j} f\right\|_{L^{2}} \\
& \leqslant \frac{1}{C_{\bar{V}}^{2} C_{\bar{V}}^{\prime 2}}\left(\left\|f^{2}\right\|_{L^{\infty}}\left\|\partial_{j} f_{\varepsilon}-\partial_{j} f\right\|_{L^{2}}+\left(\|f\|_{L^{\infty}}+\left\|f_{\varepsilon}\right\|_{L^{\infty}}\right)\left\|\left(f-f_{\varepsilon}\right) \partial_{j} f\right\|_{L^{2}}\right)
\end{aligned}
$$

Now the first term is converges to zero by Lemma 2.3(iii) and the second by dominated convergence (cf. (9) for $p=2$ ).

Now we return to the issue of nondegeneracy of the smoothings of gt-regular metrics and take a closer look at the determinant of the smoothing. Note that we have to deal with $\operatorname{det}\left(\mathbf{g}_{\varepsilon}\right)$ rather than $(\operatorname{det} \mathbf{g})_{\varepsilon}$, which means that we cannot simply use the results on the scalar case above. We again aim at some uniform control, more precisely at a condition of the form

$\forall K$ compact $\exists C_{K}^{\prime} \exists \varepsilon_{0}(K):\left|\operatorname{det}\left(\mathbf{g}_{\varepsilon}\right)\right| \geqslant C_{K}^{\prime}>0 \forall x \in K, \forall \varepsilon \leqslant \varepsilon_{0}(K)$,

since this will also imply that the smoothed metric is nondegenerate in the generalised sense. Of course if $\mathbf{g}$ was continuous then the convergence would be locally uniform and 
the determinant $\operatorname{det}\left(\mathbf{g}_{\varepsilon}\right)$ would obey (12) due to (6). However, in the general case we shall use the following stability condition for gt-regular metrics.

Definition 4.5 (Stability condition for gt-regular metrics). Let $\mathbf{g}$ be a gt-regular metric and denote by $\lambda_{i}, \ldots, \lambda_{n}$ its eigenvalues.

(i) For any compact $K$ we denote by

$$
\mu_{K}:=\min _{1 \leqslant i \leqslant n} \operatorname{essinf}\left|\lambda^{i}(x)\right|,
$$

the (essential) absolute infimum of any eigenvalue of $\mathbf{g}$ on $K$.

(ii) We call $\mathbf{g}$ stable if for each compact $K$ there is a continuous $(0,2)$-tensor field $\mathbf{A}^{K}$ on $K$ such that for $1 \leqslant j, k \leqslant n$

$$
\underset{x \in K}{\operatorname{essup}}\left|g_{i j}(x)-A_{i j}^{K}(x)\right| \leqslant C_{K}^{\prime \prime}<\frac{\mu_{K}}{2 n} .
$$

Note that if $\mathbf{g}$ is nondegenerate then $\mu_{K}>0$ for all $K$. Then the stability condition means that on compact sets the entries of the metric $\mathbf{g}$ differ from those of a continuous function by an amount proportional to the smallest eigenvalue, i.e, the entries do not vary too wildly as compared with the smallest eigenvalue. This condition seems to be quite natural as a consideration of the diagonal case shows and furthermore allows enough control on the smoothing of the metric to guarantee the eigenvalues, and hence the determinant, is bounded away from zero uniformly on compact sets for all $\varepsilon$ small. More precisely, we have.

Proposition 4.6 (Nondegeneracy of smoothed gt-regular metrics). Let $\mathrm{g}$ be $a$ nondegenerate, stable, gt-regular metric and let $\mathbf{g}_{\varepsilon}$ be a smoothing of $\mathbf{g}$ obtained by convolution with an admissible mollifier $\left(\rho_{\varepsilon}\right)_{\varepsilon}$. Then its determinant $\operatorname{det}\left(\mathbf{g}_{\varepsilon}\right)$ is uniformly nonvanishing on compact sets, i.e.,

$$
\forall K \text { compact } \exists C_{K}^{\prime} \exists \varepsilon_{0}(K):\left|\operatorname{det}\left(\mathbf{g}_{\varepsilon}(x)\right)\right| \geqslant C_{K}^{\prime}>0 \forall x \in K, \forall \varepsilon \leqslant \varepsilon_{0}(K) .
$$

In particular, the embedding $\iota_{\rho}(\mathbf{g})$ of $\mathbf{g}$ is a generalised metric.

In the proof of Proposition 4.6 we shall need the following Lemma which exploits the stability property to give a suitable uniform control on the smoothing.

Lemma 4.7 (Squeezing). Let $f \in L_{\mathrm{loc}}^{\infty}$ and let $L$ be compact. Suppose that there exists a continuous function $f^{L}$ on $L$ such that $\left\|f-f^{L}\right\|_{L^{\infty}(L)} \leqslant a_{L}$. Then we have $\forall$ compact $K \subset \subset L \forall \sigma>0 \exists \varepsilon_{0}(K, \sigma): \quad\left\|f-f_{\varepsilon}\right\|_{L^{\infty}(K)} \leqslant 2 a_{L}+\sigma \quad \forall \varepsilon \leqslant \varepsilon_{0}(K, \sigma)$.

Proof. Let $K, L$ be as in the statement and write

$\left\|f-f_{\varepsilon}\right\|_{L^{\infty}(K)} \leqslant\left\|f-f^{L}\right\|_{L^{\infty}(K)}+\left\|f^{L}-f_{\varepsilon}^{L}\right\|_{L^{\infty}(K)}+\left\|f_{\varepsilon}^{L}-f_{\varepsilon}\right\|_{L^{\infty}(K)}$.

The first term on the r.h.s. of (14) is bounded by $a_{L}$ and the second converges to zero thanks to the continuity of $f^{L}$. Finally, the third one is bounded by (2) and Lemma 4.3 (v) by

$\left\|f_{\varepsilon}^{L}-f_{\varepsilon}\right\|_{L^{\infty}(K)}=\left\|\left(f^{L}-f\right) * \rho_{\varepsilon}\right\|_{L^{\infty}(K)} \leqslant\left\|f^{L}-f\right\|_{L^{\infty}(L)}\left\|\rho_{\varepsilon}\right\|_{L^{1}} \leqslant a_{L}(1+\eta)$

for all $\varepsilon$ small enough. 
Proof of Proposition 4.6. Let $K$ be compact, choose $L$ compact with $K \subset \subset L$ and choose $\sigma$ such that $C_{L}^{\prime \prime}+\sigma / 2 \leqslant \mu_{L} /(2 n)$. Now the stability condition (13) together with Lemma 4.7 implies that $\left\|g_{i j}-g_{i j}^{\varepsilon}\right\|_{L^{\infty}(K)} \leqslant 2 C_{L}^{\prime \prime}+\sigma<\mu_{L} / n$ for all $i, j$ and all $\varepsilon$ small. Hence the maximum difference of the eigenvalues of $\mathbf{g}$ and $\mathbf{g}_{\varepsilon}$ is bounded by $\max _{1 \leqslant i \leqslant n}\left\|\lambda^{i}-\lambda_{\varepsilon}^{i}\right\|_{L^{\infty}(K)} \leqslant \operatorname{ess~sup}_{x \in K}\left\|\mathbf{g}(x)-\mathbf{g}_{\varepsilon}(x)\right\| \leqslant n \max _{1 \leqslant i, j \leqslant n}\left\|g_{i j}-g_{i j}^{\varepsilon}\right\|_{L^{\infty}(K)}<\mu_{L} \leqslant \mu_{K}$, where \|\| denotes any suitable matrix norm. By definition of $\mu_{K}$ the modulus $\left|\lambda_{\varepsilon}^{i}\right|$ of all eigenvalues of $\mathbf{g}_{\varepsilon}$ is uniformly bounded from below on $K$ for $\varepsilon$ small enough and so is the determinant.

Using the result on the determinant we are finally in a position to look at the stability of the inverse of the smoothed metric. In particular, we have.

Proposition 4.8 (Stability of the inverse). Let $\mathbf{g}$ be a nondegenerate, stable, gt-regular metric and let $\mathbf{g}_{\varepsilon}$ be a smoothing of $\mathbf{g}$ obtained by convolution with an admissible mollifier $\rho_{\varepsilon}$. Then for any open and relatively compact $V$ there exists $\varepsilon_{0}(V)$ such that the inverse of the smoothing $\left(\mathbf{g}_{\varepsilon}\right)^{-1}$ is a smooth and uniformly bounded net on $V$ for all $\varepsilon \leqslant \varepsilon_{0}(V)$ and we have

$$
\left(\mathbf{g}_{\varepsilon}\right)^{-1} \rightarrow \mathbf{g}^{-1} \text { in } H_{\mathrm{loc}}^{1} \cap L_{\mathrm{loc}}^{p} \text { for all } p<\infty .
$$

In particular, for any embedding $\iota_{\rho}$ we have that $\left(\iota_{\rho}(\mathbf{g})\right)^{-1} \approx \mathbf{g}^{-1}$.

Proof. By Proposition $4.6\left|\operatorname{det}\left(\mathbf{g}_{\varepsilon}\right)\right|$ is locally uniformly bounded away from zero on compact sets hence the components of the inverse of the smoothed metric $g_{\varepsilon}^{i j}:=$ $\left(\left(\mathbf{g}_{\varepsilon}\right)^{-1}\right)_{i j}=\operatorname{cof} g_{i j}^{\varepsilon} / \operatorname{det}\left(\mathbf{g}_{\varepsilon}\right)$ form a smooth and uniformly bounded net on any open, relatively compact $V$. To prove the statement on convergence we first write for $p<\infty$

$$
\begin{aligned}
\left\|g_{\varepsilon}^{i j}-g^{i j}\right\|_{L^{p}(V)} & =\left\|\frac{\operatorname{cof} g_{i j}^{\varepsilon}}{\operatorname{det}\left(\mathbf{g}_{\varepsilon}\right)}-\frac{\operatorname{cof} g_{i j}}{\operatorname{det} \mathbf{g}}\right\|_{L^{p}}=\left\|\frac{\operatorname{cof} g_{i j}^{\varepsilon} \operatorname{det} \mathbf{g}-\operatorname{cof} g_{i j} \operatorname{det}\left(\mathbf{g}_{\varepsilon}\right)}{\operatorname{det} \mathbf{g} \operatorname{det}\left(\mathbf{g}_{\varepsilon}\right)}\right\|_{L^{p}} \\
\leqslant & \left\|\frac{1}{\operatorname{det} \mathbf{g} \operatorname{det}\left(\mathbf{g}_{\varepsilon}\right)}\right\|_{L^{\infty}}\left(\left\|\operatorname{cof} g_{i j}^{\varepsilon} \operatorname{det} \mathbf{g}-\operatorname{cof} g_{i j}^{\varepsilon} \operatorname{det}\left(\mathbf{g}_{\varepsilon}\right)\right\|_{L^{p}}\right. \\
& \left.+\left\|\operatorname{cof} g_{i j}^{\varepsilon} \operatorname{det}\left(\mathbf{g}_{\varepsilon}\right)-\operatorname{cof} g_{i j} \operatorname{det}\left(\mathbf{g}_{\varepsilon}\right)\right\|_{L^{p}}\right) \\
& \frac{1}{C_{\bar{V}} C_{\bar{V}}^{\prime}}\left(\left\|\operatorname{cof} g_{i j}^{\varepsilon}\right\|_{L^{\infty}}\left\|\operatorname{det} \mathbf{g}-\operatorname{det}\left(\mathbf{g}_{\varepsilon}\right)\right\|_{L^{p}}+\left\|\operatorname{det}\left(\mathbf{g}_{\varepsilon}\right)\right\|_{L^{\infty}}\left\|\operatorname{cof} g_{i j}^{\varepsilon}-\operatorname{cof} g_{i j}\right\|_{L^{p}}\right),
\end{aligned}
$$

where the respective first terms are bounded by (2) and convergence is due to Proposition 4.2.

To prove $H_{\text {loc }}^{1}$-convergence we write

$$
\begin{gathered}
\left\|\partial_{l}\left(g_{\varepsilon}^{i j}-g^{i j}\right)\right\|_{L^{2}(V)} \\
=\left\|\frac{\left(\partial_{l} \operatorname{cof} g_{i j}^{\varepsilon}\right) \operatorname{det}\left(\mathbf{g}_{\varepsilon}\right)-\operatorname{cof} g_{i j}^{\varepsilon} \partial_{l} \operatorname{det}\left(\mathbf{g}_{\varepsilon}\right)}{\left(\operatorname{det}\left(\mathbf{g}_{\varepsilon}\right)\right)^{2}}-\frac{\left(\partial_{l} \operatorname{cof} g_{i j}\right) \operatorname{det} \mathbf{g}-\operatorname{cof} g_{i j} \partial_{l} \operatorname{det} \mathbf{g}}{(\operatorname{det} \mathbf{g})^{2}}\right\|_{L^{2}} \\
\leqslant\left\|\frac{1}{\operatorname{det} \mathbf{g} \operatorname{det}\left(\mathbf{g}_{\varepsilon}\right)}\right\|_{L^{\infty}}\left(\left\|\operatorname{det} \mathbf{g} \partial_{l} \operatorname{cof} g_{i j}^{\varepsilon}-\operatorname{det} \mathbf{g} \partial_{l} \operatorname{cof} g_{i j}\right\|_{L^{2}}\right. \\
\left.+\left\|\operatorname{det} \mathbf{g} \partial_{l} \operatorname{cof} g_{i j}-\operatorname{det}\left(\mathbf{g}_{\varepsilon}\right) \partial_{l} \operatorname{cof} g_{i j}\right\|_{L^{2}}\right)
\end{gathered}
$$




$$
\begin{gathered}
+\left\|\frac{1}{\left(\operatorname{det} \mathbf{g} \operatorname{det}\left(\mathbf{g}_{\varepsilon}\right)\right)^{2}}\right\|_{L^{\infty}}\left(\left\|(\operatorname{det} \mathbf{g})^{2} \operatorname{cof} g_{i j}^{\varepsilon} \partial_{l} \operatorname{det}\left(\mathbf{g}_{\varepsilon}\right)-(\operatorname{det} \mathbf{g})^{2} \operatorname{cof} g_{i j}^{\varepsilon} \partial_{l} \operatorname{det} \mathbf{g}\right\|_{L^{2}}\right. \\
+\left\|(\operatorname{det} \mathbf{g})^{2} \operatorname{cof} g_{i j}^{\varepsilon} \partial_{l} \operatorname{det} \mathbf{g}-(\operatorname{det} \mathbf{g})^{2} \operatorname{cof} g_{i j} \partial_{l} \operatorname{det} \mathbf{g}\right\|_{L^{2}} \\
\left.+\left\|(\operatorname{det} \mathbf{g})^{2} \operatorname{cof} g_{i j} \partial_{l} \operatorname{det} \mathbf{g}-\left(\operatorname{det}\left(\mathbf{g}_{\varepsilon}\right)\right)^{2} \operatorname{cof} g_{i j} \partial_{l} \operatorname{det} \mathbf{g}\right\|_{L^{2}}\right) \\
\leqslant C\left(\left\|\partial_{l} \operatorname{cof} g_{i j}^{\varepsilon}-\partial_{l} \operatorname{cof} g_{i j}\right\|_{L^{2}}+C\left\|\partial_{l} \operatorname{cof} g_{i j}\left(\operatorname{det}\left(\mathbf{g}_{\varepsilon}\right)-\operatorname{det} \mathbf{g}\right)\right\|_{L^{2}}\right) \\
+C\left(\left\|\operatorname{cof} g_{i j}^{\varepsilon}\right\|_{L^{\infty}}\left\|\partial_{l} \operatorname{det}\left(\mathbf{g}_{\varepsilon}\right)-\partial_{l} \operatorname{det} \mathbf{g}\right\|_{L^{2}}+\left\|\left(\partial_{l} \operatorname{det} \mathbf{g}\right)\left(\operatorname{cof} g_{i j}^{\varepsilon}-\operatorname{cof} g_{i j}\right)\right\|_{L^{2}}\right. \\
\left.\quad+C\left\|\operatorname{cof} g_{i j}\right\|_{L^{\infty}}\left\|\partial_{l} \operatorname{det} \mathbf{g}\left((\operatorname{det} \mathbf{g})^{2}-\left(\operatorname{det}\left(\mathbf{g}_{\varepsilon}\right)\right)^{2}\right)\right\|_{L^{2}}\right) .
\end{gathered}
$$

Now the first and third term converges to zero by Proposition 4.2 and the bound from (2), while for the other terms we again use dominated convergence as in (9).

Finally, we have a corresponding statement on the convergence of the Christoffel symbols.

Proposition 4.9 (Stability of the Christoffel symbols). Let $\mathbf{g}$ be a nondegenerate, stable, gt-regular metric and let $\mathbf{g}_{\varepsilon}$ be a smoothing of $\mathbf{g}$ obtained by convolution with an admissible mollifier $\left(\rho_{\varepsilon}\right)_{\varepsilon}$. Then for any open and relatively compact $V$ there exists $\varepsilon_{0}(V)$ such that the Christoffel symbols of the first and of the second kind of the smoothing $\Gamma_{i j k}\left[\mathbf{g}_{\varepsilon}\right]$ and $\Gamma_{j k}^{i}\left[\mathbf{g}_{\varepsilon}\right]$ are smooth and $L^{2}$-bounded nets on $V$ for $\varepsilon \leqslant \varepsilon_{0}(V)$ and we have

$$
\Gamma_{i j k}\left[\mathbf{g}_{\varepsilon}\right] \rightarrow \Gamma_{i j k} \text { and } \Gamma_{j k}^{i}\left[\mathbf{g}_{\varepsilon}\right] \rightarrow \Gamma_{j k}^{i} \text { in } L_{\mathrm{loc}}^{2}
$$

In particular, for any embedding $\iota_{\rho}$ we have

$$
\Gamma_{i j k}\left[\iota_{\rho}(\mathbf{g})\right] \approx \Gamma_{i j k}[\mathbf{g}] \text { and } \Gamma_{j k}^{i}\left[\iota_{\rho}(\mathbf{g})\right] \approx \Gamma_{j k}^{i}[\mathbf{g}] .
$$

Proof. Smoothness of the $\Gamma_{i j k}\left[\mathbf{g}_{\varepsilon}\right]$ is clear and $L^{2}(V)$-boundedness follows from estimate (2) together with the fact that convolution commutes with taking derivatives.

For the $\Gamma_{j k}^{i}\left[\mathbf{g}_{\varepsilon}\right]$ smoothness follows from the smoothness statement on the inverse in Proposition 4.8 whereas $L^{2}(V)$-boundedness follows as above and taking into account the $L^{\infty}(V)$-boundedness of the inverse, again given in Proposition 4.8.

As for convergence the statement on $\Gamma_{i j k}\left[\mathbf{g}_{\varepsilon}\right]$ simply follows from Lemma 2.3 (iii) and again the fact that the derivative of the smoothing is the smoothing of the derivative.

For $\Gamma_{j k}^{i}\left[\mathbf{g}_{\varepsilon}\right]$ observe that we have to deal with a sum of terms of the form $g_{\varepsilon}^{i j} \partial_{l} g_{r s}^{\varepsilon}=g_{\varepsilon}^{i j}\left(\partial_{l} g_{r s}\right)_{\varepsilon}$ which due to Proposition 4.8 are precisely of the form covered in Lemma 4.1 (ii) with $p=2$ and $m=1$.

\section{Compatibility results}

We have now collected all prerequisites to precisely state our main result, saying that the Geroch-Traschen approach to distributional metrics is compatible with the Colombeau approach. 
Theorem 5.1 (Compatibility for the Riemann curvature). Let $\mathbf{g}$ be a nondegenerate, stable, gt-regular metric and denote its Riemann tensor by Riem $[\mathbf{g}]$. Let $\mathbf{g}_{\varepsilon}$ be a smoothing of $\mathbf{g}$ obtained by convolution with an admissible mollifier $\left(\rho_{\varepsilon}\right)_{\varepsilon}$. Then we have for the Riemann tensor Riem $\left[\mathbf{g}_{\varepsilon}\right]$ of $\mathbf{g}_{\varepsilon}$

$$
\operatorname{Riem}\left[\mathbf{g}_{\varepsilon}\right] \rightarrow \operatorname{Riem}[\mathbf{g}] \text { in } \mathcal{D}^{\prime} .
$$

In other words, for any embedding $\iota_{\rho}(\mathbf{g})$ of $\mathbf{g}$ we have

$$
\operatorname{Riem}\left[\iota_{\rho}(\mathbf{g})\right] \approx \operatorname{Riem}[g] .
$$

Before giving the proof, which using the results of the previous section is fairly short, we illustrate the content of the theorem in a diagram.

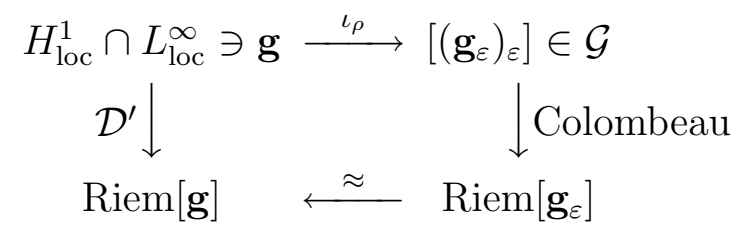

Given a nondegenerate, stable and gt-regular metric $\mathbf{g}$ we can either derive the Riemann curvature Riem $[\mathbf{g}]$ in distributions or embed $\mathbf{g}$ via convolution with an admissible mollifier to obtain the generalised metric $\left[\left(\mathbf{g}_{\varepsilon}\right)\right]$. If we then derive its curvature Riem $\left[\mathbf{g}_{\varepsilon}\right]$ within the generalised setting we find that it is associated with the distributional curvature Riem $[\mathbf{g}]$.

Proof of Theorem 5.1. In coordinates we have

$$
R_{j k l}^{i}\left[\mathbf{g}_{\varepsilon}\right]=\partial_{l} \Gamma_{k j}^{i}\left[\mathbf{g}_{\varepsilon}\right]-\partial_{k} \Gamma_{l j}^{i}\left[\mathbf{g}_{\varepsilon}\right]+\Gamma_{l m}^{i}\left[\mathbf{g}_{\varepsilon}\right] \Gamma_{k j}^{m}\left[\mathbf{g}_{\varepsilon}\right]-\Gamma_{k m}^{i}\left[\mathbf{g}_{\varepsilon}\right] \Gamma_{l j}^{m}\left[\mathbf{g}_{\varepsilon}\right]
$$

Now by Proposition $4.9 \Gamma_{j k}^{i}\left[\mathbf{g}_{\varepsilon}\right] \rightarrow \Gamma_{j k}^{i}[\mathbf{g}]$ in $L_{\text {loc }}^{2}$ hence in $\mathcal{D}^{\prime}$ and we obtain $\partial_{l} \Gamma_{j k}^{i}\left[\mathbf{g}_{\varepsilon}\right] \rightarrow \partial_{l} \Gamma_{j k}^{i}[\mathbf{g}]$ in distributions. By continuity of the product $L_{\mathrm{loc}}^{2} \times L_{\mathrm{loc}}^{2} \rightarrow L_{\mathrm{loc}}^{1}$ we obtain $\Gamma_{j k}^{i}\left[\mathbf{g}_{\varepsilon}\right] \Gamma_{r s}^{l}\left[\mathbf{g}_{\varepsilon}\right] \rightarrow \Gamma_{j k}^{i}[\mathbf{g}] \Gamma_{r s}^{l}[\mathbf{g}]$ in $L_{\mathrm{loc}}^{1}$, hence again in distributions.

Similarly we also have compatibility results for the Ricci, Weyl and scalar curvature. To prepare for the formulation and proof of these results we recall from [1] that it is possible to define the outer product of (any number of copies of inverses of) a gt-regular metric with its Riemann tensor. Indeed in the smooth case we may write $\frac{1}{2} g^{r s} R_{j k l}^{i}=g^{r s}\left(\partial_{[l} \Gamma_{k] j}^{i}+g^{r s} \Gamma_{m[l}^{i} \Gamma_{k] j}^{m}\right)=\partial_{[l}\left(g^{r s} \Gamma_{k] j}^{i}\right)-\left(\partial_{[l} g^{r s}\right) \Gamma_{k] j}^{i}+g^{r s} \Gamma_{m[l}^{i} \Gamma_{k] j}^{m}$,

and we see that the right hand side makes sense in distributions for a gt-regular metric. Indeed, $g^{r s} \Gamma_{k j}^{i} \in L_{\text {loc }}^{2}$ allows for a weak derivative as well as $\left(\partial_{l} g^{r s}\right) \Gamma_{k j}^{i} \in L_{\mathrm{loc}}^{1} \ni g^{r s} \Gamma_{m l}^{i} \Gamma_{k j}^{m}$. Moreover, the same holds true for any product of the form $\otimes_{m} \mathbf{g} \otimes_{l} \mathbf{g}^{-1} \otimes \operatorname{Riem}[\mathbf{g}]$ : just use the Leibnitz rule on $\partial_{l}\left(\otimes_{m} \mathbf{g} \otimes_{l} \mathbf{g}^{-1} \Gamma_{j k}^{i}\right)$. We now have.

Corollary 5.2 (Compatibility for curvature quantities). Let $\mathrm{g}$ be a nondegenerate, stable, gt-regular metric and let $\mathbf{g}_{\varepsilon}$ be a smoothing of $\mathbf{g}$ obtained by convolution with an admissible mollifier $\left(\rho_{\varepsilon}\right)_{\varepsilon}$. Then we have $(m, l \in \mathbb{N})$

$$
\otimes_{m} \mathbf{g}_{\varepsilon} \otimes_{l} \mathbf{g}_{\varepsilon}^{-1} \otimes \operatorname{Riem}\left[\mathbf{g}_{\varepsilon}\right] \rightarrow \otimes_{m} \mathbf{g} \otimes_{l} \mathbf{g}^{-1} \otimes \operatorname{Riem}[g] \text { in } \mathcal{D}^{\prime} .
$$


In particular, the result applies to the Ricci, Weyl and scalar curvature and with other words we have for any embedding $\iota_{\rho}(\mathbf{g})$ of $\mathbf{g}$

$$
\operatorname{Ric}\left[\iota_{\rho}(\mathbf{g})\right] \approx \operatorname{Ric}[\mathbf{g}], W\left[\iota_{\rho}(\mathbf{g})\right] \approx W[\mathbf{g}], R\left[\iota_{\rho}(\mathbf{g})\right] \approx R[\mathbf{g}] .
$$

Proof. According to the above discussion we have to deal with the terms

$$
\partial_{l}\left(g_{i_{1} j_{1}}^{\varepsilon} \cdots g_{i_{m} j_{m}}^{\varepsilon} g_{\varepsilon}^{r_{1} s_{1}} \cdots g_{\varepsilon}^{r_{l} s_{l}} \Gamma_{j k}^{i}\left[\mathbf{g}_{\varepsilon}\right]\right), \partial_{l}\left(g_{i_{1} j_{1}}^{\varepsilon} \cdots g_{i_{m} j_{m}}^{\varepsilon} g_{\varepsilon}^{r_{1} s_{1}} \cdots g_{\varepsilon}^{r_{l} s_{l}}\right) \Gamma_{j k}^{i}\left[\mathbf{g}_{\varepsilon}\right]
$$

and

$$
g_{i_{1} j_{1}}^{\varepsilon} \cdots g_{i_{m} j_{m}}^{\varepsilon} g_{\varepsilon}^{r_{1} s_{1}} \cdots g_{\varepsilon}^{r_{l} s_{l}} \Gamma_{m j}^{i}\left[\mathbf{g}_{\varepsilon}\right] \Gamma_{l j}^{m}\left[\mathbf{g}_{\varepsilon}\right] .
$$

To deal with the first one note that

$$
g_{i_{1} j_{1}}^{\varepsilon} \cdots g_{i_{m} j_{m}}^{\varepsilon} g_{\varepsilon}^{r_{1} s_{1}} \cdots g_{\varepsilon}^{r_{l} s_{l}} \Gamma_{j k}^{i}\left[\mathbf{g}_{\varepsilon}\right] \rightarrow g_{i_{1} j_{1}} \cdots g_{i_{m} j_{m}} g^{r_{1} s_{1}} \cdots g^{r_{l} s_{l}} \Gamma_{j k}^{i}[\mathbf{g}]
$$

in $L_{\text {loc }}^{2}$ by Lemma 4.1(ii) for $p=2$, hence in distributions and we obtain the desired convergence of the derivatives. For the second term note that by the Leibnitz rule we only have to show that

$g_{i_{1} j_{1}}^{\varepsilon} \cdots\left(\partial_{l} g_{i_{p} j_{p}}^{\varepsilon}\right) \cdots g_{i_{m} j_{m}}^{\varepsilon} g_{\varepsilon}^{r_{1} s_{1}} \cdots g_{\varepsilon}^{r_{l} s_{l}} \Gamma_{j k}^{i}\left[\mathbf{g}_{\varepsilon}\right] \rightarrow g_{i_{1} j_{1}} \cdots\left(\partial_{l} g_{i_{p} j_{p}}\right) \cdots g_{i_{m} j_{m}} g^{r_{1} s_{1}} \cdots g^{r_{l} s_{l}} \Gamma_{j k}^{i}[\mathbf{g}]$

(and analogously for the terms with the derivative falling on the inverse). However, this holds true in $L_{\text {loc }}^{1}$, hence $\mathcal{D}^{\prime}$ by Lemma 4.1(ii) and by continuity of the product $L_{\mathrm{loc}}^{2} \times L_{\mathrm{loc}}^{2} \rightarrow L_{\mathrm{loc}}^{1}$. Finally, the same argument applies to $g_{i_{1} j_{1}}^{\varepsilon} \cdots g_{i_{m} j_{m}}^{\varepsilon} g_{\varepsilon}^{r_{1} s_{1}} \cdots g_{\varepsilon}^{r_{l} s_{l}} \Gamma_{m j}^{i}\left[\mathbf{g}_{\varepsilon}\right] \Gamma_{l j}^{m}\left[\mathbf{g}_{\varepsilon}\right]$.

Finally, we discuss the relation of our results to the stability results obtained by Geroch and Traschen in [1] and LeFloch and Mardare in [3]. To begin with we remark that in their Theorem 4.6, LeFloch and Mardare [3] suppose convergence of $\mathbf{g}_{\varepsilon}^{-1}$ to $\mathrm{g}^{-1}$ in $L_{\mathrm{loc}}^{\infty}$ which is not true in case of smoothings via convolution unless the metric is supposed to be more regular, e.g. continuous. In this case our result coincides with theirs while in general we deal with nets that converge only in a weaker sense.

On the other hand the relation with the results of Geroch and Traschen is more subtle. Theorem 2 of [1] asserts that for any sequence of gt-regular metrics $\mathbf{g}_{n}$ that is $L_{\mathrm{loc}}^{\infty}$-bounded together with its inverse $\left(\mathbf{g}_{n}\right)^{-1}$ and for which $\mathbf{g}_{n},\left(\mathbf{g}_{n}\right)^{-1}$ and $\partial_{i} \mathbf{g}_{n}$ converge in $L_{\text {loc }}^{2}$ to $\mathbf{g}, \mathbf{g}^{-1}$ resp. $\partial_{i} \mathbf{g}$ the sequence Riem $\left[\mathbf{g}_{n}\right]$ of Riemann tensors converges to Riem $[\mathbf{g}]$ in $\mathcal{D}^{\prime}$. Actually, in the context of the present work, the nondegenerate and stability conditions we impose on a gt-regular metric ensure that the conditions required for their Theorem 2 are satisfied as a consequence of our Lemma 2.3 together with Propositions 4.8 and 4.9. Thus our Theorem 5.1 follows from [1, Thm. 2] but we feel that our proof is more direct. Indeed a mild variation of our proof provides a simpler proof of their Theorem.

Also note that our results on the stability of the inverse metric and the Christoffel symbols are more precise and actually provide the best possible $W_{\text {loc }}^{m, p}$-convergence: If we had converge in any smaller $W_{\text {loc }}^{m, p}$-space then by completeness the original metric would have had to be in that space too. 
Finally, we note that Theorem 4 in [1] shows that for any continuous, gt-regular metric there is a sequence of smooth metrics (actually obtained by smoothing via convolution) which converges in the above mentioned sense. However, recall from the discussion preceding Definition 4.5 that the question of nondegeneracy in the continuous case is much easier to handle. The question of whether the requirement for continuity could be omitted from the assumptions was left open in [1] with the proof failing to cover this case. Our results provide a positive answer to this question: For any nondegenerate, stable, gt-regular metric the smoothing provides a smooth sequence which converges in the desired sense.

\section{Acknowledgments}

This work was supported by projects P16742, P20525, and Y-237 of the Austrian Science Fund.

\section{Appendix A. The existence of suitable mollifiers}

As pointed out in Section 2.3 above one crucial feature of the Colombeau approach is that the space $\mathcal{C}^{\infty}$ of smooth functions is a subalgebra of the algebra of generalised functions $\mathcal{G}$. This is achieved by the fact that the embeddings $\iota$ and $\sigma$ coincide for smooth functions, i.e., $\sigma(f)-\iota(f) \in \mathcal{N}$ for all smooth $f$. The crucial estimate (cf. [17, Prop. 1.2.11]) in turn is based on the fact that the mollifier used to define $\iota$ is assumed to have vanishing moments. It is actually this requirement that forces us to assume $\rho \in \mathcal{S}$ since there do not exist any compactly supported smooth functions with all moments vanishing. Moreover, a function with all moments vanishing can never be nonnegative and also has an infinite number of zeroes. It is this property which makes it a nontrivial task to preserve positivity when embedding distributions into $\mathcal{G}$. One solution to this problem is discussed in this appendix.

The key step in our approach is to replace the embedding $\iota$ by convolution with a suitable strict $\delta$-net $\left(\rho_{\varepsilon}\right)_{\varepsilon}$ which eventually has vanishing moments and has negative part with arbitrarily small $L^{1}$-norm. Since we are now convolving with a strict $\delta$-net rather than a model $\delta$-net, i.e., a net obtained by scaling a single function $\varphi$, we have to be careful to obtain moderateness of $\left(u * \psi_{\varepsilon}\right)_{\varepsilon}$ (cf. [17, Prop. 1.2.10]). The latter property will be a consequence of moderateness of $\left(\rho_{\varepsilon}\right)_{\varepsilon}$ itself. We start by providing a suitable net $\left(\psi_{\varepsilon}\right)_{\varepsilon}$ : the scaled version denoted by $\left(\rho_{\varepsilon}\right)_{\varepsilon}$ being the admissible mollifiers used in section 4 .

Lemma Appendix A.1 (Existence of suitable mollifiers). There exists a net $\left(\psi_{\varepsilon}\right)_{\varepsilon}$ of test functions on $\mathbb{R}^{n}$ with the properties

(i) $\operatorname{supp}\left(\psi_{\varepsilon}\right) \subseteq B_{1}(0)$ for all $\varepsilon \in(0,1]$

(ii) $\int \psi_{\varepsilon}(x) d x=1 \quad$ for all $\varepsilon \in(0,1]$ 
(iii) $\forall \alpha \in \mathbb{N}_{0}^{n} \exists p: \sup _{x \in \mathbb{R}^{n}}\left|\partial^{\alpha} \psi_{\varepsilon}(x)\right|=O\left(\varepsilon^{-p}\right)$

(iv) $\forall j \in \mathbb{N} \exists \varepsilon_{0}: \int x^{\alpha} \psi_{\varepsilon}(x) d x=0 \quad$ for all $1 \leqslant|\alpha| \leqslant j$ and all $\varepsilon \leqslant \varepsilon_{0}$

(v) $\forall \eta>0 \exists \varepsilon_{0}: \int\left|\psi_{\varepsilon}(x)\right| d x \leqslant 1+\eta \quad$ for all $\varepsilon \leqslant \varepsilon_{0}$.

In particular,

$$
\rho_{\varepsilon}:=\frac{1}{\varepsilon^{n}} \psi_{\varepsilon}\left(\frac{\dot{\varepsilon}}{\varepsilon}\right)
$$

is a strict $\delta$-net, which is moderate, has finally vanishing moments and its negative parts have arbitrarily small $L^{1}$-norm, i.e., $\rho_{\varepsilon}$ satisfies (iii)-(v).

This statement can actually be proved by an application of [27, Thm. 3.10] along the lines of [27, Props. 5.1, 5.2]. However, since this reference uses the language of "internal sets" - a concept inspired by nonstandard analysis (for related work see also [28]) — we have chosen to include a direct proof.

Proof. We will be concerned with the following sets $\left(m \in \mathbb{N}_{0}, \eta>0\right)$

$\mathcal{A}_{m}:=\left\{\varphi \in \mathcal{D}\left(\mathbb{R}^{n}\right): \operatorname{supp}(\varphi) \subseteq B_{1}(0), \int \varphi=1, \int x^{\alpha} \varphi(x) d x=0 \quad \forall 1 \leqslant|\alpha| \leqslant m\right\}$,

$\mathcal{A}_{m}^{\prime}(\eta):=\left\{\varphi \in \mathcal{A}_{m}: \int|\varphi| \leqslant 1+\eta\right\}$.

It is well known that the sets $\mathcal{A}_{m} \neq \emptyset$ (see e.g. [17, Pro. 1.4.2]; the additional requirement on the supports asserted here is easily obtained by scaling). Following [27, Prop. 5.1.] we now prove that also the sets

$$
\mathcal{A}_{m}^{\prime}(\eta) \neq \emptyset, \quad \text { for all } m \in \mathbb{N}_{0} \text { and all } \eta>0 .
$$

It suffices to prove the result in the 1-dimensional case $n=1$ : the general case then follows by taking tensor products of functions of one variable. We proceed by induction. $\underline{m=0}: \mathcal{A}_{m}^{\prime}(\eta) \neq \emptyset$ even for $\eta=0$, since it suffices to choose $0 \leqslant \varphi \in \mathcal{D}(\mathbb{R})$ with $\operatorname{supp}(\varphi) \subseteq B_{1}(0)$ and $\int \varphi=1$.

$\underline{m-1 \mapsto m}$ : Let $\varphi \in \mathcal{A}_{m-1}^{\prime}(\eta / 2)$ and set $\psi:=a \varphi+b \varphi(. / \mu)$, where $a, b$, and $0<\mu<1$ are to be specified below. We have

$$
\int \psi=a+b \mu, \int x^{k} \psi(x) d x=0 \quad \forall 1 \leqslant k \leqslant m-1,
$$

as well as

$$
\int x^{m} \psi(x) d x=\left(a+b \mu^{m+1}\right) \int x^{m} \varphi(x) d x .
$$

Solving $a+b \mu=1$ and $a+b \mu^{m+1}=0$ for $a$ and $b$ we obtain

$$
a=\frac{-\mu^{m}}{1-\mu^{m}}(<0) \text { and } b=\frac{1}{\mu-\mu^{m+1}}(>0)
$$


and so

$$
\int|\psi| \leqslant(|a|+|b| \mu) \int|\varphi| \leqslant \frac{1+\mu^{m}}{1-\mu^{m}}\left(1+\frac{\eta}{2}\right)
$$

which can be made smaller than $1+\eta$ if $\mu$ is chosen small enough. So we obtain $\psi \in \mathcal{A}_{m}^{\prime}(\eta)$ and we are done.

Now we choose

$$
\varphi_{m} \in \mathcal{A}_{m}^{\prime}(1 / m) \text { and set } M_{m}:=\sup _{x \in \mathbb{R}^{n},|\alpha| \leqslant m}\left|\partial^{\alpha} \varphi_{m}(x)\right|
$$

and define the sets

$$
\mathcal{A}_{m, \varepsilon}:=\left\{\varphi \in \mathcal{A}_{m}^{\prime}(1 / m): \sup _{x \in \mathbb{R}^{n},|\alpha| \leqslant m}\left|\partial^{\alpha} \varphi(x)\right| \leqslant \frac{1}{\varepsilon}\right\} .
$$

Note that by the above $\mathcal{A}_{m, \varepsilon} \neq \emptyset$ if $\varepsilon \leqslant 1 / M_{m}=: \varepsilon_{0}(m)$ and $\mathcal{A}_{m+1, \varepsilon} \subseteq \mathcal{A}_{m, \varepsilon}$ for all $\varepsilon$. Now for $m \in \mathbb{N}, \varepsilon \leqslant \varepsilon_{0}(m)$ we choose $\psi_{m, \varepsilon} \in \mathcal{A}_{m, \varepsilon}$ and finally set

$$
\psi_{\varepsilon}:=\psi_{m, \varepsilon} \quad \varepsilon_{0}(m+1)<\varepsilon \leqslant \varepsilon_{0}(m) .
$$

We then obviously have (i) and (ii) and it remains to verify (iii)-(v).

(iii): Let $|\alpha| \in \mathbb{N}_{0}^{n}$. Then since $\psi_{\varepsilon} \in \mathcal{A}_{|\alpha|, \varepsilon}$ for $\varepsilon \leqslant \varepsilon_{0}(|\alpha|)$ we obtain $\sup _{x \in \mathbb{R}^{n}}\left|\partial^{\alpha} \psi_{\varepsilon}(x)\right| \leqslant$ $1 / \varepsilon$ for all $\operatorname{such} \varepsilon$.

(iv): Let $|\alpha| \geqslant 1$. Then since $\psi_{\varepsilon} \in \mathcal{A}_{|\alpha|}$ for $\varepsilon \leqslant \varepsilon_{0}(|\alpha|)$ we have $\int x^{\alpha} \psi_{\varepsilon}(x) d x=0$ for all such $\varepsilon$.

(v): Let $\eta>0$ and choose $m$ such that $1 / m \leqslant \eta$. Since $\psi_{\varepsilon} \in \mathcal{A}_{m}^{\prime}(1 / m)$ for all $\varepsilon \leqslant \varepsilon_{0}(m)$ we have for all such $\varepsilon$ that $\int\left|\psi_{\varepsilon}\right| \leqslant 1+1 / m \leqslant 1+\eta$.

Finally we observe that the mollifiers obtained above in fact provide an embedding of distributions into the Colombeau algebra.

Corollary Appendix A.2 (An embedding of distributions). Let $u \in \mathcal{D}^{\prime}\left(\mathbb{R}^{n}\right)$ and let $\left(\rho_{\varepsilon}\right)_{\varepsilon}$ be a strict $\delta$-net as in Lemma Appendix A.1. Then the mapping

$$
\iota_{\rho}: u \mapsto\left[\left(u * \rho_{\varepsilon}\right)_{\varepsilon}\right]
$$

is a linear embedding of $\mathcal{D}^{\prime}\left(\mathbb{R}^{n}\right)$ into $\mathcal{G}\left(\mathbb{R}^{n}\right)$ having the distinguishing properties

(i) $\iota_{\rho} \circ \partial^{\alpha}=\partial^{\alpha} \circ \iota_{\rho} \quad$ for all $\alpha \in \mathbb{N}_{0}^{n}$

(ii) $\left.\iota_{\rho}\right|_{\mathcal{C}^{\infty}}=\sigma$

(iii) $\iota_{\rho}(u) \approx u$

(iv) $\iota_{\rho}$ preserves supports.

Proof. The proof is just a mild variation of the usual "standard proofs". So we only remark that for proving moderateness of $\iota_{\rho}(u)$ as well as for proving (ii) and (iv) ((i) and (iii) follow directly from the properties of the convolution) we just have to use moderateness of $\left(\rho_{\varepsilon}\right)_{\varepsilon}$ in the respective proofs of Propositions 1.2.10-1.2.12 in [17]. 


\section{References}

[1] Geroch R and Traschen J 1987 Strings and other distributional sources in general relativity Phys. Rev. D 36 1017-1031

[2] Garfinkle D 1999 Metrics with distributional curvature Class. Quant. Grav. 16 4101-4109

[3] LeFloch P and Madare C 2007 Definition and stability of lorentzian manifolds with distributional curvature Port. Math. (N.S.) 64 535-573

[4] Steinbauer R 2008 A note on distributional semi-riemannian geometry Preprint

[5] Colombeau J F 1984 New Generalized Functions and Multiplication of Distributions (Amsterdam: North Holland)

[6] Colombeau J F 1985 Elementary Introduction to New Generalized Functions (Amsterdam: North Holland)

[7] Colombeau J F 1990 Multiplication of distributions Bull. Amer. Math. Soc. (N.S.) 23 251-268

[8] Clarke C J S, Vickers J A and Wilson J P 1996 Generalised functions and distributional curvature of cosmic strings Class. Quant. Grav. 13 2485-2498

[9] Vickers J A and Wilson J P 2000 Generalized hyperbolicity in conical spacetimes Class. Quantum. Grav. 17 1333-1360

[10] Balasin H 1997 Distributional energy-momentum tensor of the extended Kerr geometry Class. Quant. Grav. 14 3353-3362

[11] Balasin H 1997 Geodesics for impulsive gravitational waves and the multiplication of distributions Class. Quant. Grav. 14 455-462

[12] Kunzinger M and Steinbauer R 1999 A rigorous solution concept for geodesic and geodesic deviation equations in impulsive gravitational waves J. Math. Phys. 40 1479-1489

[13] Grant J D E, Mayerhofer E and Steinbauer R 2009 The wave equation on singular space times Commun. Math. Phys. (to appear)

[14] Steinbauer R and Vickers J A 2006 The use of generalized functions and distributions in general relativity Classical Quantum Gravity 23 R91-R114

[15] Marsden J E 1968 Generalized Hamiltonian mechanics Arch. Rat. Mech. Anal. 28 323-361

[16] Parker P 1979 Distributional geometry J. Math. Phys. 20 1423-1426

[17] Grosser M, Kunzinger M, Oberguggenberger M and Steinbauer R 2001 Geometric Theory of Generalized Functions (Mathematics and its Applications vol 537) (Dordreacht: Kluwer)

[18] Evans L 1998 Partial differential equations (Graduate Studies in Mathematics vol 19) (Providence, RI: American Mathematical Society)

[19] Kunzinger M and Steinbauer R 2002 Foundations of a nonlinear distributional geometry Acta Appl. Math. 71 179-206

[20] Delcroix A 2005 Remarks on the embedding of spaces of distributions into spaces of colombeau generealized functions Novi Sad J. Math. 35 27-40

[21] Grosser M, Kunzinger M, Steinbauer R and Vickers J 2002 A global theory of algebras of generalized functions Adv. Math. 166 179-206

[22] Grosser M, Kunzinger M, Steinbauer R and Vickers J A 2008 A global theory of algebras of generalized functions II: tensor distributions Preprint

[23] Schwartz L 1954 Sur l'impossibilité de la multiplication des distributions C. R. Acad. Sci. Paris 239 847-848

[24] Kunzinger M and Steinbauer R 2002 Generalized pseudo-Riemannian geometry. Trans. Amer. Math. Soc. 354 4179-4199

[25] Oberguggenberger M 1992 Multiplication of Distributions and Applications to Partial Differential Equations (Pitman Research Notes in Mathematics vol 259) (Harlow: Longman Scientific and Technical)

[26] Adams R A 1975 Sobolev Spaces (New York: Academic Press)

[27] Vernaeve $\mathrm{H}$ and Oberguggenberger M 2008 Internal sets and internal functions in colombeau theory J. Math. Anal. Appl. 341 649-659 
[28] Oberguggenberger M and Todorov T 1998 An embedding of Schwartz distributions in the algebra of asymptotic functions Int. J. Math. Math. Sci. 21 417-428 